\title{
Twenty years of physics at MAMI — What did it mean?
}

\author{
B.A. Meckinga \\ Thomas Jefferson National Accelerator Facility, 12000 Jefferson Avenue, Newport News, VA 23606, USA \\ / \\ Published online: 7 June 2006 - C Società Italiana di Fisica / Springer-Verlag 2006
}

\begin{abstract}
The development over the last twenty years of the physics program and the experimental facilities at the Mainz Microtron MAMI will be reviewed. Ground-breaking contributions have been made to the development of experimental techniques and to our understanding of the structure of nucleons and nuclei.
\end{abstract}

PACS. 29.17. $+\mathrm{w}$ Electrostatic, collective, and linear accelerators - 25.20.-x Photonuclear reactions 25.30.Bf Elastic electron scattering - 25.30.Dh Inelastic electron scattering to specific states

\section{Introduction}

The goal of nuclear physics is to study the properties of nuclei, and to understand these properties on the basis of the fundamental theory of the constituents making up the nucleus. Quantum-chromodynamics (QCD) has emerged as the leading candidate for the theory of hadronic interactions. Presently, QCD cannot be solved in the strongcoupling regime due to the lack of appropriate perturbative solution. The best hope in the near future is to use large-scale numerical calculations to approximate the space-time continuum by a discrete lattice in the framework of Lattice QCD (LQCD). Due to limitations in compute power, the lattice spacing is still fairly coarse, and the masses of the quarks used are much larger than their actual values. These limitations require significant extrapolations which need to be constrained theoretically.

For zero-mass quarks, QCD can be solved in a rigorous way via Chiral Perturbation Theory $(\chi \mathrm{PT})$. Again, an extrapolation is required, this time from zero-mass quarks up to the actual values. An interesting recent development is the use of functional forms derived from $\chi \mathrm{PT}$ to extrapolate LQCD results.

Before these extrapolations can be trusted, it is very important to verify the predictive power of $\chi \mathrm{PT}$ via experimental tests, in particular via pion photoproduction close to threshold.

On the experimental side, valuable information on the properties of bound quark systems is still lacking. In particular, the knowledge of the spatial distribution of the charges and the currents inside the nucleon is not satisfactory, especially for the neutron. Our knowledge of the excited states of the nucleon is still insufficient, e.g. what are the degrees-of-freedom governing the mass spectrum,

\footnotetext{
a e-mail: mecking@jlab.org
}

and what are the differences in quark wave functions between the ground state and the excited states.

Many of the issues mentioned above can be investigated using electron scattering. Electrons interact only with the charged constituents of the object under investigation. The interaction is described by Quantum-Electrodynamics (QED), and is sufficiently small to be handled with perturbative methods.

In the past, the usefulness of the electromagnetic probe was limited by the technical features of the available electron accelerators. In particular, coincidence experiments and the operation of large acceptance detectors were hampered by the low duty-cycle of the electron beams. Dramatic progress in accelerator and detector technology has made it possible to overcome these limitations and to study electromagnetic processes with an accuracy that is no longer limited by technical problems.

The Institut für Kernphysik (Institute for Nuclear Physics) at the University of Mainz in Germany has been at the forefront of this development. For more than 20 years, the Institute has developed novel electron accelerators (the MAMI series of microtrons) and the corresponding experimental equipment, and has used those devices for ground-breaking research into the electromagnetic structure of nucleons and nuclei.

On the occasion of the retirement of six key people (Hartmuth Arenhövel, Hartmut Backe, Dieter Drechsel, Jörg Friedrich, Karl-Heinz Kaiser, and Thomas Walcher) from the Institute, a symposium was held in October 2005 to review 20 years of Physics at MAMI and to commemorate their contributions.

This paper will attempt to review the major contributions MAMI and its user community have made to the field, to identify the particular circumstances that made these contributions possible, and to speculate on their lasting impact. 


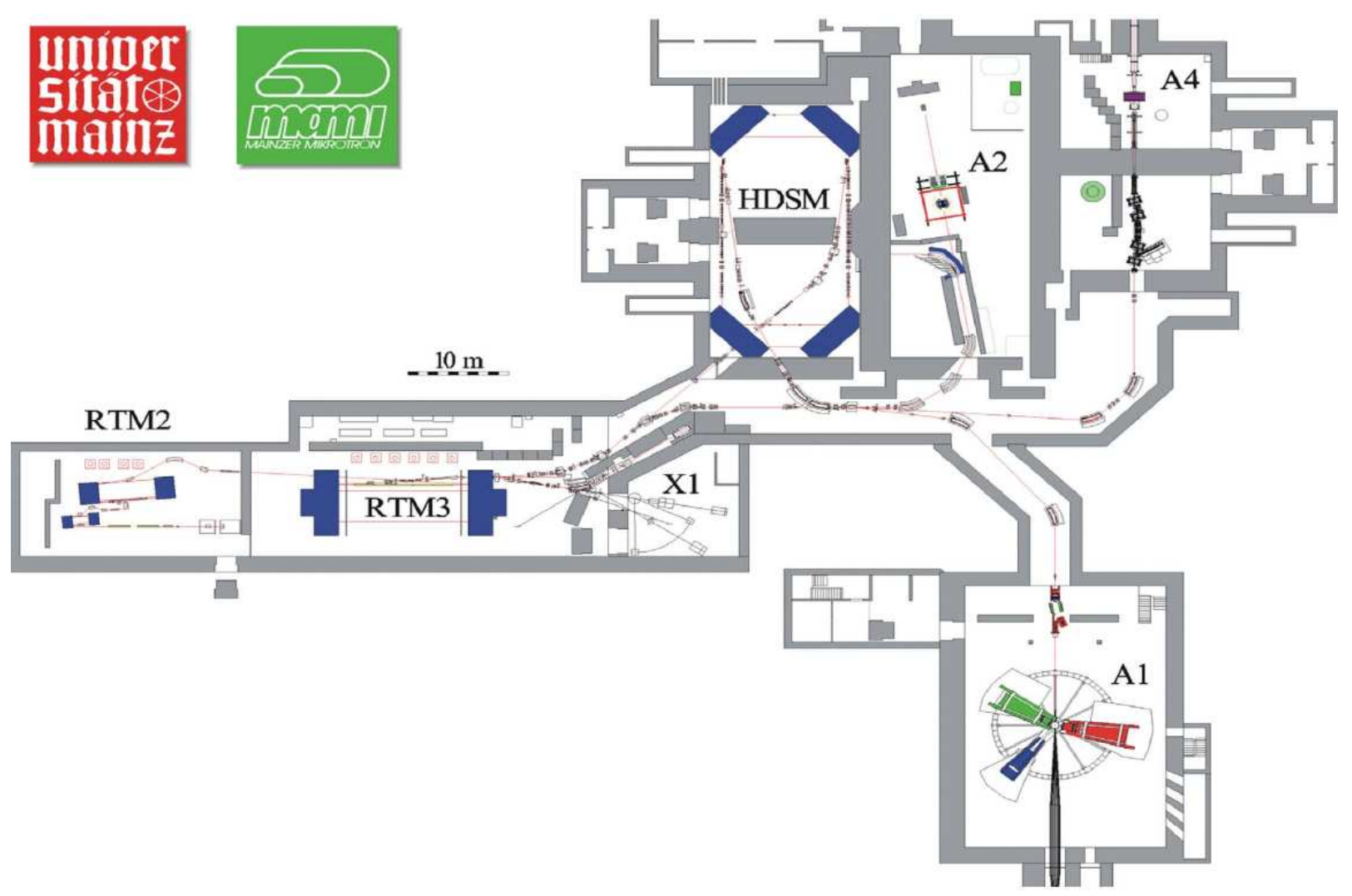

Fig. 1. Layout of the MAMI accelerators and the experimental areas. MAMI A is located in the RTM2 area, MAMI B in RTM3, the new MAMI C in the area labeled HDSM.

Table 1. MAMI microtron development.

\begin{tabular}{|c|c|}
\hline Year & Activity \\
\hline 1975 & $\begin{array}{l}\text { Proposal for a Race-Track Microtron } \\
\text { (design by H. Herminghaus et al.) }\end{array}$ \\
\hline 1979 & $14 \mathrm{MeV}$ beam from MAMI A1 \\
\hline 1982 & $\begin{array}{l}\text { Preliminary Sonderforschungsbereich } \\
\text { (SFB) established }\end{array}$ \\
\hline 1983 & $183 \mathrm{MeV}$ beam from MAMI A2 \\
\hline $1983-87$ & MAMI A operation with a total of $18,700 \mathrm{~h}$ \\
\hline $1983-90$ & Development of the $855 \mathrm{MeV}$ MAMI B \\
\hline 1984 & SFB 201 established \\
\hline 1990 & $\begin{array}{l}\text { First } 855 \mathrm{MeV} \text { beam from MAMI B } \\
\text { (first experiment by A2 Collaboration) }\end{array}$ \\
\hline $1990-2005$ & MAMI B operation with a total of $82,843 \mathrm{~h}$ \\
\hline 1999 & Sonderforschungsbereich 443 established \\
\hline 2000 & $\begin{array}{l}\text { Approval of } 1.5 \mathrm{GeV} \text { HDSM } \\
\text { (Harmonic Double-Sided Microtron, } \\
\text { design by K.-H. Kaiser et al.) }\end{array}$ \\
\hline $2001-03$ & Installation of the four HDSM magnets \\
\hline 2006 & Commissioning and begin of physics \\
\hline
\end{tabular}

\section{MAMI microtron development}

The history of the MAMI microtron development is summarized in table 1 . The pioneering development of MAMI $\mathrm{A}$ and $\mathrm{B}$ at Mainz has established the microtron as a cost- effective way to build an accelerator capable of delivering a high quality electron beam [1]. The layout of the accelerator and its experimental areas is shown in fig. 1. The microtron design relies on sending the beam repeatedly through the same room-temperature accelerating structure with moderate energy gain per turn. Recirculation is achieved by two homogeneous $180^{\circ}$ end-magnets. The size of these end-magnets for the last microtron stage, MAMI $\mathrm{B}$, is evident from fig. 2. The perpendicular entry and exit of the electron orbits at the end-magnets results in simple and robust beam optics. Due to the continuous-wave nature of the radio-frequency power and the constant magnetic field, the quality of the beam is very high: an energy spread of $\delta E / E=1.5 \times 10^{-5}$ and an emittance of $\epsilon=8 \times$ $10^{-9} \mathrm{~m}$ is achieved routinely. A laser-driven polarized gun produces electron beams with $80 \%$ polarization. Parity violation experiments are possible since helicity-correlated changes in the beam parameters are very small: energy variations of $\delta E / E \leq 10^{-8}$ and position variations of $\delta x \leq$ $100 \mathrm{~nm}$ have been achieved. Particularly impressive is the high operational stability: overnight and during weekends, the MAMI microtrons are routinely operated by students.

It has been the conventional wisdom in the accelerator community that the maximum energy of a microtron is limited to about one $\mathrm{GeV}$ since the construction of the end-magnets which increase rapidly in size with increasing energy becomes technically and financially impractical. 


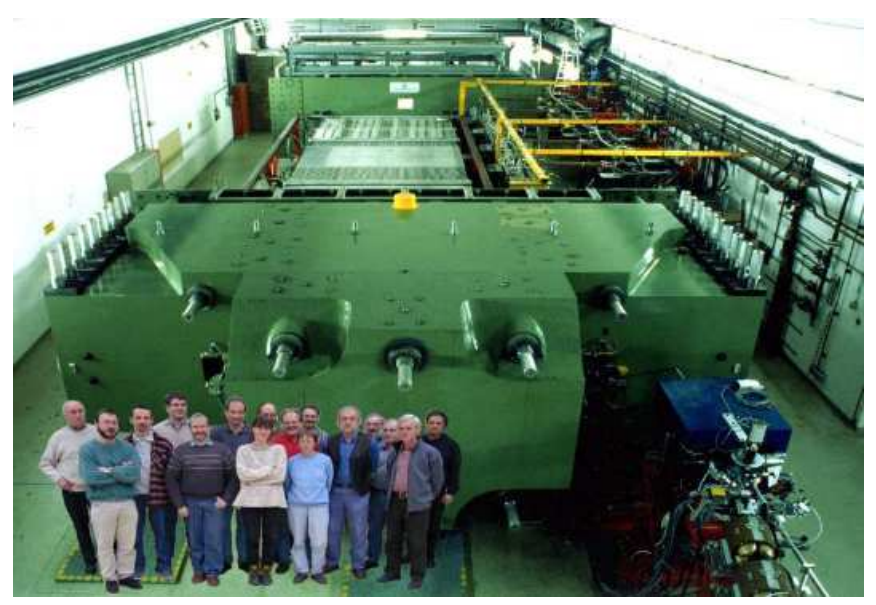

Fig. 2. The MAMI accelerator team standing in front of one of the MAMI B $180^{\circ}$ end-magnets. The common accelerating section is located between the magnets on the right-hand side, the separated return paths are on the left-hand side.

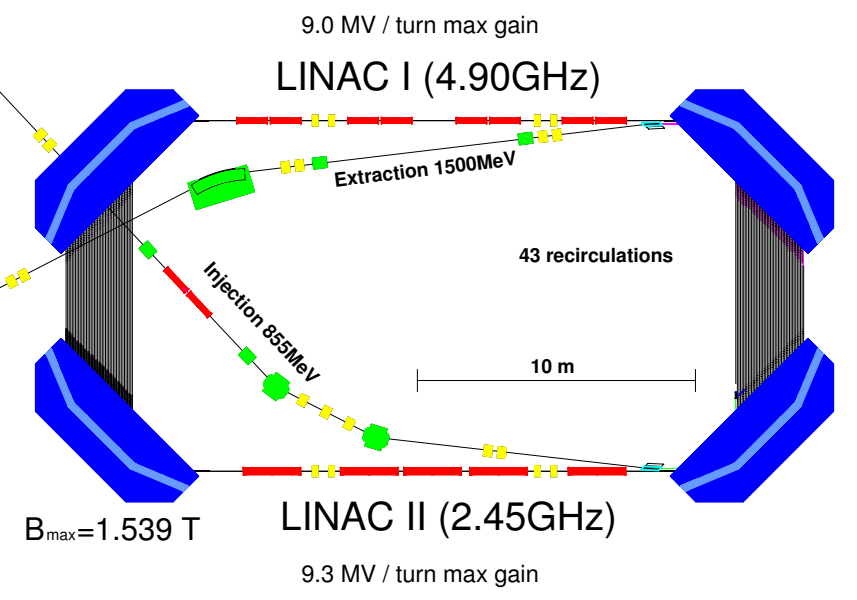

Fig. 3. Layout of the MAMI C microtron.

The design and construction of the $1.5 \mathrm{GeV}$ Harmonic Double-Sided Microtron (HDSM, design by K.-H. Kaiser et al.) [2] is poised to shatter that boundary. Building on the experience with the previous microtrons, the challenging HDSM design relies on two parallel accelerating sections joined by four inhomogeneous $90^{\circ}$ end-magnets with a weight of 250 metric tons each (see fig. 3 for a layout). The strong vertical defocusing at the entrance and exit of the magnets is compensated by a radial gradient field. Meeting the microtron coherence condition within the confined space of the existing experimental area forces the fundamental accelerating frequency to be twice the frequency of the MAMI B microtron. Phase stability considerations require to leave one of the two accelerating sections at the present MAMI B frequency. The installation of the four HDSM magnets has been completed, and commissioning is expected to start soon.

As shown in table 1, important milestones parallel to the technical developments were the establishment of the "Sonderforschungsbereiche" ("Special Research Ini-
Table 2. Three-spectrometer system parameters.

\begin{tabular}{lrrr}
\hline Spectrometer & $\mathrm{A}$ & $\mathrm{B}$ & $\mathrm{C}$ \\
\hline configuration & QSDD & $\mathrm{D}$ & QSDD \\
$p_{\max }[\mathrm{MeV} / c]$ & 665 & 810 & 490 \\
$\delta \Omega[\mathrm{msr}]$ & 28 & 5.6 & 28 \\
$\Theta_{\min }$ & $18^{\circ}$ & $7^{\circ}$ & $18^{\circ}$ \\
$\delta p / p[\%]$ & 20 & 15 & 25 \\
\hline
\end{tabular}

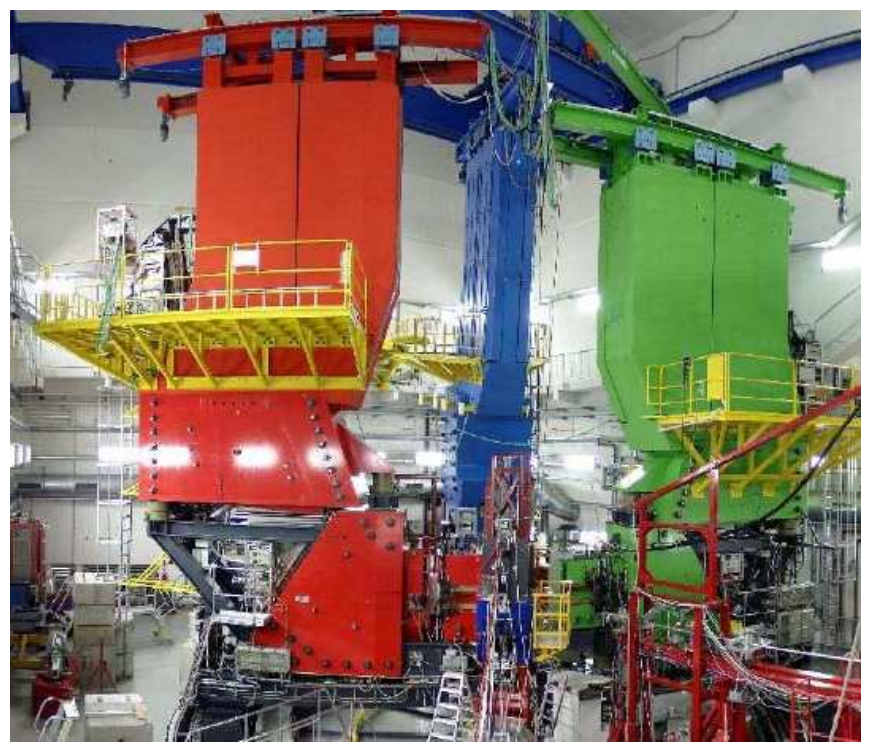

Fig. 4. Photograph of the three high-resolution magnetic spectrometers in the A1 area. Spectrometer A (red) is on the lefthand side, B (blue) in the center, and C (green) is on the right-hand side.

tiatives", abbreviated SFB), a funding scheme used by the German funding agency Deutsche Forschungsgemeinschaft (DFG) to support new initiatives for a limited period of time.

\section{Experimental equipment at MAMI}

The broad physics program at MAMI requires an equally broad range of experimental equipment, from high-resolution magnetic spectrometers to large acceptance detectors. Most of the electron scattering instrumentation has been provided by the Institute, a large fraction of the equipment for the tagged photon experiments has been contributed by the user community. For electron scattering experiments, the three-spectrometer system offers an unprecedented combination of momentum resolution, solid angles, and momentum range. The parameters are given in table 2, a photograph is shown in fig. 4 .

For experiments with real photons, the Glasgow-Mainz bremsstrahlung tagging system, located in the A2 area, provides photons of known energy and flux. Circularly polarized photons can be obtained from the bremsstrahlung of polarized electrons, linearly polarized photons from an oriented crystal radiator. The detection equipment is focused on charged and neutral particle detection in a large 


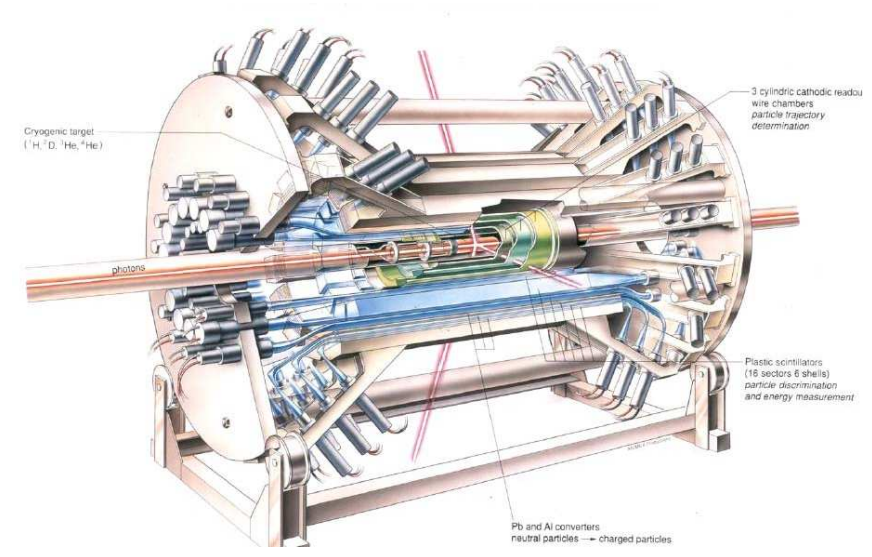

Fig. 5. The DAPHNE large acceptance detector.

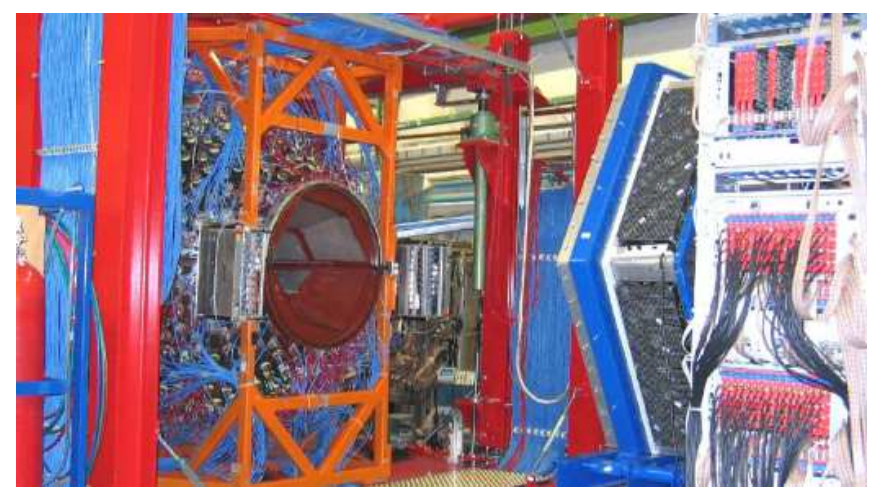

Fig. 6. The Crystal Ball detector (left) and TAPS (right).

solid angle and energy range. The Saclay-built DAPHNE detector (Détecteur à grande Acceptance pour la Physique photo-Nucléaire Expérimentale) uses a combination of proportional wire chambers and layers of scintillation counters and absorbers for charged particle and photon detection. A three-dimensional drawing is shown in fig. 5.

The TAPS detector (original abbreviation for TwoArm Photon Spectrometer) can be arranged in different configurations. Its $528 \mathrm{BaF}_{2}$ crystals give good energy resolution for photon detection. Charged particles can be identified via the ratio of fast and slow scintillation light.

The newest addition to the experimental arsenal is the Crystal Ball detector which has seen prior service at highenergy facilities like SPEAR, DORIS, and the BNL AGS. Its central detector consists of $672 \mathrm{NaI}$ crystals, again optimized for photon detection. The electronic readout system has been modernized and equipped with $80 \mathrm{MHz}$ flashADC's. In the first experiment, the Crystal Ball will be used in combination with TAPS as a forward detector (see fig. 6 for a picture of the setup) to measure the $\Delta^{+}$magnetic moment via the angular distribution of the decay photons in the $\Delta^{+} \rightarrow \Delta^{+} \gamma$ transition (from the high-mass tail of the $\Delta^{+}$to its low-mass tail).

Of particular importance for studying the spin degreesof-freedom of the nucleon has been the addition of the Bonn frozen-spin polarized $\mathrm{H}$ and $\mathrm{D}$ target (see fig. 7 for a picture). Its low magnetic holding field and open geometry make it an ideal match for large acceptance detectors.

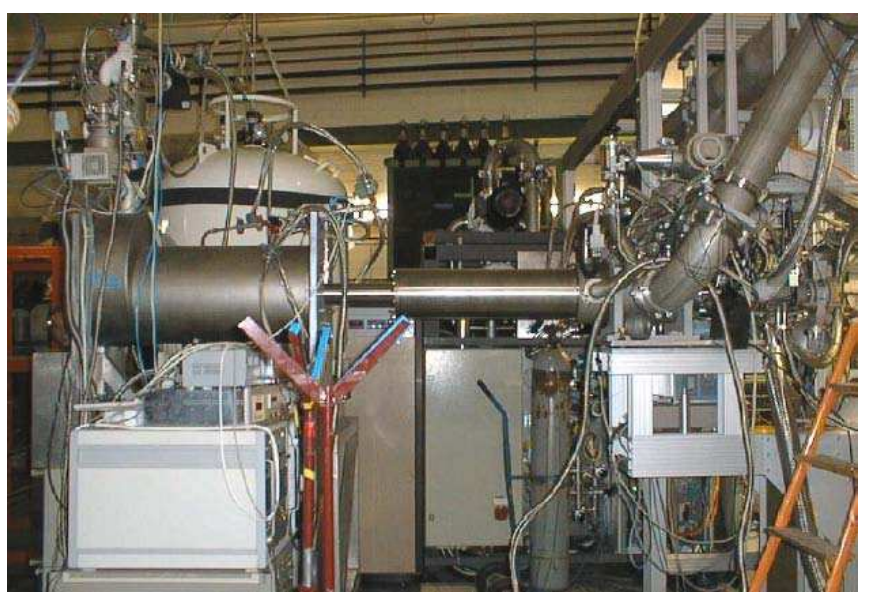

Fig. 7. The Bonn frozen-spin polarized target during a repolarization cycle. The polarizing magnet is on the left-hand side, the pumping unit on the right-hand side.

In combination with the DAPHNE detector it has been used for studying the GDH sum rule on hydrogen and deuterium.

\section{Selected experiments}

The following sections will give examples for experiments that are characteristic for the MAMI physics program and that have had a large impact on the field.

\subsection{Real Compton Scattering and the Polarizability of the Proton}

The electric and magnetic polarizabilities of the nucleon are static quantities that characterize the response of the system to external electric and magnetic fields. Since the highest fields that one can produce in the laboratory are much too weak to have a measurable influence, the best approach is to derive the polarizabilities from the energy and angular dependence of real photon scattering (RCS) at low photon energies. The experiments are challenging since the cross sections are very small and, above pion production threshold, there is a large background of photons from $\pi^{0}$ decays.

The experimental results obtained with the TAPS detector and the bremsstrahlung tagging system [3] require significant theoretical corrections and interpretation (see fig. 8 for an example).

The cross section is dominated by scattering off the charge and the magnetic moment of the proton. In addition, the incident and outgoing photons can couple to an exchanged pion. Finally, the polarizabilities enter linearly only in the low-energy expansion. In practice, higher-order terms need to be incorporated. A dispersion relation analysis [4] of the entire body of Compton scattering data shows that the proton is a very "stiff" objects, i.e. it does not deform much under the influence of external static fields. 


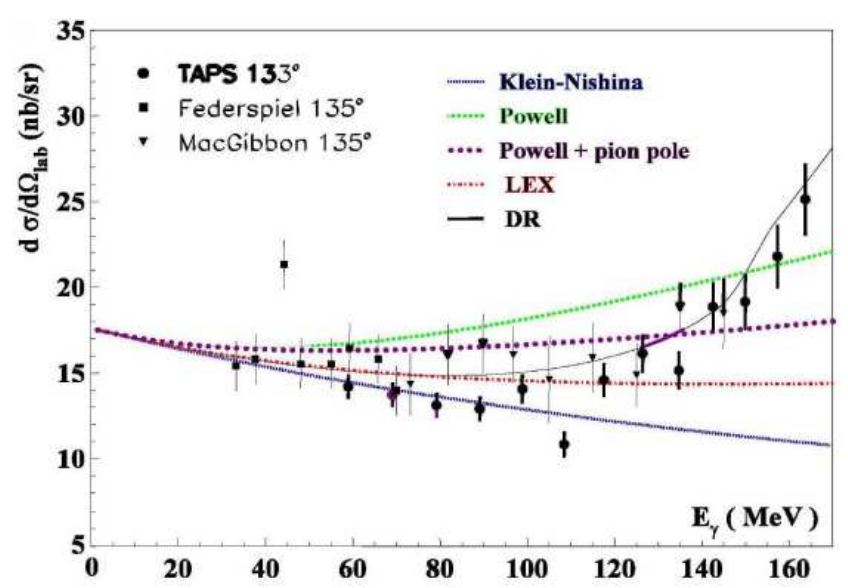

Fig. 8. Photon energy dependence of the Compton scattering cross section at a scattering angle of $135^{\circ}$. The dispersion relation analysis is the thin black line marked DR.

\subsection{Generalized nucleon polarizabilities and virtual Compton scattering}

The concept of the nucleon polarizabilities can be extended to virtual photons. This leads to six general polarizabilities which are functions of the momentum transfer, $Q$, and can be accessed in the $e p \rightarrow e^{\prime} p(\gamma)$ reaction. The differential cross section is dominated by the Bethe-Heitler process and by Born terms which can be calculated from QED and the known proton form factors. These contributions have to be subtracted from the measured cross sections.

At MAMI, the process was measured by using two high-resolution magnetic spectrometers to detect the scattered electron and the recoiling proton, and using the missing-mass technique to identify the (undetected) photon in the final state [5].

From the MAMI data (see fig. 9), the generalized polarizabilities $\alpha_{E}\left(Q^{2}\right)$ and $\beta_{M}\left(Q^{2}\right)$ have been determined using a dispersion theoretical analysis [4]. The data are well described by taking the asymptotic and the $\pi N$-contribution into account; this demonstrates the importance of the pion cloud contribution to the nucleon polarizability.

\subsection{Near-threshold $\pi^{0}$ production}

The physics goal of this program is to test the predictions of $\chi \mathrm{PT}$ which is an exact representation of QCD for the limiting case $m_{\pi} \rightarrow 0$. To compare to the real world, an extrapolation to physical pion mass required. This introduces low-energy constants into $\chi \mathrm{PT}$ which need to be determined from experiment. Once the low-energy constants are known, the predictive power of $\chi \mathrm{PT}$ can be tested.

The experimental information required are precise $\pi^{0}$ and $\pi^{+}$photo- and electro-production differential cross sections close to threshold where $\chi \mathrm{PT}$ is expected to be valid. In addition, polarization data are necessary to separate the contributing multipoles. The experimental challenges are considerable: the cross sections are small, and

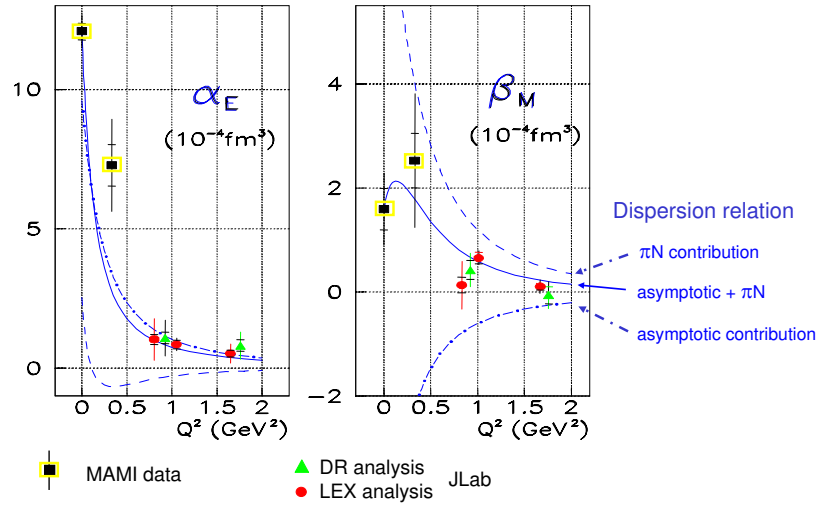

Fig. 9. $Q^{2}$-dependence of the generalized polarizabilities $\alpha_{E}\left(Q^{2}\right)$ and $\beta_{M}\left(Q^{2}\right)$.

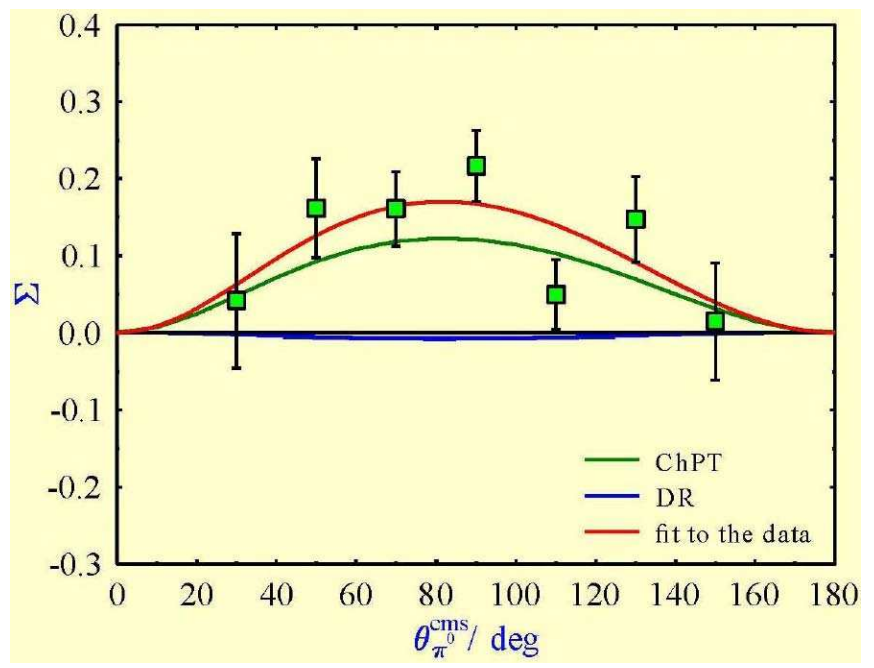

Fig. 10. Beam asymmetry for $\pi^{0}$ photoproduction as a function of the $\pi^{0}$ production angle.

they have very strong photon energy dependence. Also, the reaction close to threshold typically involves low-range particles which are difficult to detect.

The experimental setup for the $\pi^{0}$-photoproduction part of the program [3] used the tagged photon beam and the TAPS photon spectrometer. Using an oriented crystal as a radiator, linearly polarized photons were produced to get the desired polarization information from the beam asymmetry. The TAPS crystals were arranged into 7 independent arrays covering the entire $\pi^{0}$ angular range. As an example for the results, fig. 10 shows the beam asymmetry as a function of the $\pi^{0}$ c.m.s. angle.

The experimental results show that $\chi \mathrm{PT}$ has considerable predictive power. This finding, in combination with other tests of $\chi \mathrm{PT}$, has increased dramatically the confidence in the validity of $\chi \mathrm{PT}$. The most important application in other areas is the use of $\chi \mathrm{PT}$ for extrapolating LQCD calculations.

The compute power required for LQCD calculations depends on the pion mass as $m_{\pi}^{-7}$. Therefore, the 


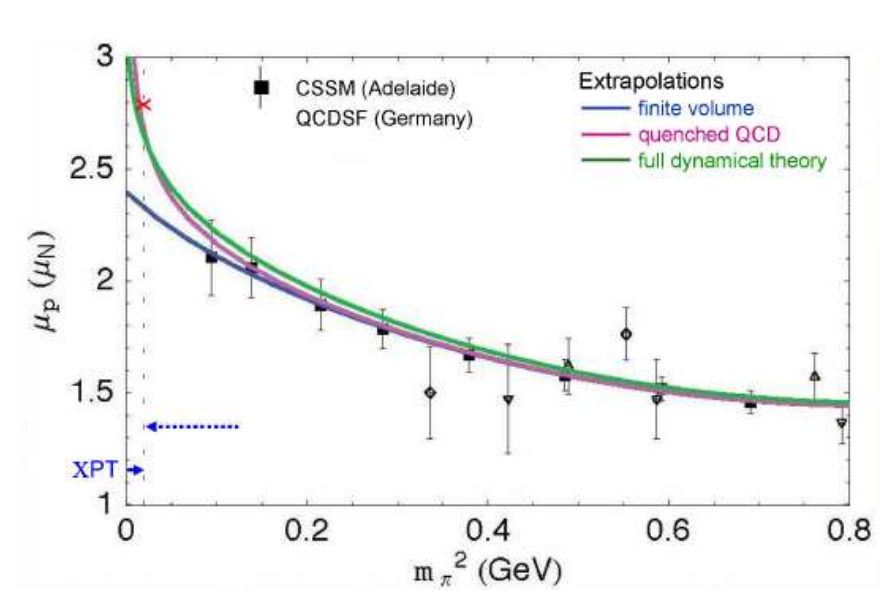

Fig. 11. LQCD results for the proton magnetic moment. The data points give the results of the calculations for different values of $m_{\pi}{ }^{2}$. The physical values of $\mu_{p}$ and $m_{\pi}{ }^{2}$ are marked by the red cross. The short blue arrow indicates the typical $\chi \mathrm{PT}$ extrapolation range; the dotted blue arrow indicates the typical LQCD extrapolation range. The best theoretical extrapolation is given by the green solid line.

calculations are performed far away from the physical pion mass. The generally accepted technique to extrapolate to the physical pion mass is to use the functional form for the $m_{\pi}$-dependence given by $\chi \mathrm{PT}$. As an example, fig. 11 shows the result of a LQCD calculation [6] for the magnetic moment of the proton as a function of $m_{\pi}^{2}$. With the chiral extrapolation, the LQCD result gets close to the true value.

\subsection{Experimental test of the GDH sum rule}

The GDH sum rule for the nucleon was derived in 1966 by Gerasimov and, independently, by Drell and Hearn. The sum rule is based on fundamental assumptions: Lorentz and gauge invariance, unitarity, and no-subtraction dispersion relations. The sum rule links the weighted integral over $\sigma_{3 / 2}(k)-\sigma_{1 / 2}(k)$ to the anomalous magnetic moment of the nucleon. $\sigma_{3 / 2}(k)$ and $\sigma_{1 / 2}(k)$ are the total hadronic photoproduction cross sections for total helicity $3 / 2$ and $1 / 2$, respectively, as a function of the photon energy $k$. The integral over $k$ extends from pion production threshold to infinity, the weight factor is $1 / k$.

This fundamental sum rule had never been tested due to the lack of appropriate experimental facilities. The test of the sum rule requires a circularly polarized photon beam and a longitudinally polarized target, both with high polarization. In addition, a detector to measure the total cross section reliably needs to be available.

A major effort has been launched in Europe to test the validity of the GDH sum rule. For the proton, the lowenergy part of the sum $(200-800 \mathrm{MeV})$ has been measured at MAMI, the contribution between 800 and $2900 \mathrm{MeV}$ has been investigated at the ELSA accelerator in Bonn. Contributions below $200 \mathrm{MeV}$ (that are difficult to access

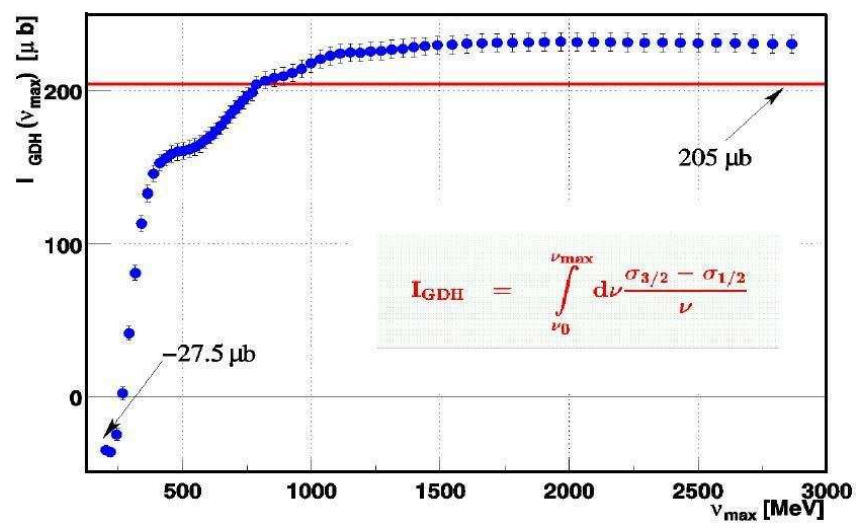

Fig. 12. GDH integral as a function of the upper limit $\nu_{\max }$.

Table 3. GDH Results from ELSA and MAMI.

\begin{tabular}{lll}
\hline Source of Information & $k[\mathrm{MeV}]$ & $I_{G D H}[\mu \mathrm{b}]$ \\
\hline MAMI & $200-800$ & $226 \pm 5 \pm 12$ \\
$\quad$ PRL 87 (2001) 022003 & & \\
ELSA & $800-2900$ & $27.5 \pm 2.0 \pm 1.2$ \\
$\quad$ PRL 93 (2004) 032003 & & \\
Low-energy extrapolation & $140-200$ & $-27.5 \pm 3$ \\
$\quad$ MAID (2003) & & \\
High-energy extrapolation & $2900-\infty$ & -14 \\
$\quad$ PLB 450 (1999) 439 & & \\
\hline Experimental sum & & $212 \pm 5 \pm 12$ \\
\hline Theoretical GDH integral & & 205 \\
\hline
\end{tabular}

experimentally) and above $2900 \mathrm{MeV}$ were estimated theoretically and added to the experimental sum.

For the MAMI GDH experiment [7], circularly polarized tagged photons from the bremsstrahlung of polarized electrons were hitting a longitudinally polarized frozenspin target. The DAPHNE detector in combination with a forward scintillation counter and shower detector was used to measure the total cross section via identifying and adding up the individual reaction channels. In addition to testing the GDH sum rule, this experimental technique also allows the measurement of the energy and angular dependence of the helicity-separated differential cross sections for the individual reaction channels that contribute to the total cross section.

From the MAMI and ELSA data, the GDH integral has been calculated. It is shown as a function of the upper integration limit in fig. 12. Table 3 gives the partial integral for different energy ranges; in the table, the statistical error is given first, the second entry is the systematic error. Also listed are the contributions from photon energies below and above the range that was covered experimentally. Note that, because of the $1 / k$-weighting, the contribution from threshold to $200 \mathrm{MeV}$ is about the same as the contribution from 800 to $2900 \mathrm{MeV}$. Within the errors, the final experimental value agrees well with the theoretical prediction, thus verifying the GDH sum rule.

First preliminary data from a polarized deuteron target are available. Extracting the GDH sum for the neutron 
from these experimental data is a conceptual challenge since the GDH sum for the deuteron is dominated by the $\gamma D \rightarrow p n$ process close to the $2.2 \mathrm{MeV}$ threshold. Only with major theoretical support will there be a chance to extract the GDH integral for the neutron from the deuteron data.

It is instructive to look at the impact the GDH effort has had on the community beyond just validating the GDH sum rule. To address this important physics question, a coherent effort of several European groups was required, an experience that will likely lead to more collaboration in the future. On the physics side, thinking in terms of the sum rule created an awareness that there are no isolated physics problems: all energy regions are interrelated, sometimes in ways that are not immediately obvious. An important consequence of the technical advances required for the GDH sum rule tests is that the measurement of helicity observables has now become routine. These observables contain valuable information, e.g. on the excitation of the nucleon resonances. Beyond the total cross section, detailed information on the energy and angular dependence for the different decay channels will be required to make the best use of the information.

\subsection{Nucleon electromagnetic form factors}

In elastic electron scattering, the response of a composite system is given by its electromagnetic form factors. These describe the probability that the system will stay intact after absorbing a virtual photon characterized by the four-momentum transfer $Q$. Form factors provide the ideal meeting ground for experiment and theory. Electric (due to the charges) and magnetic (due to the magnetization) form factors can be separated by measuring the angular dependence of the scattering cross section at constant $Q^{2}$ (Rosenbluth separation). An intuitive picture of the nucleon is provided by the spatial distributions of charge and magnetization which can be obtained from the form factors via a Fourier transform.

Separating the electric, $G_{e}$, and magnetic, $G_{m}$, form factors using the Rosenbluth separation technique is difficult when $G_{e} / G_{m} \ll 1$ which is always the case for the neutron. The solution is to use polarization transfer to measure the $G_{e} / G_{m}$ ratio. In practice, there are two possibilities: using polarized electrons on a polarized target, or using polarized electrons on an unpolarized target and analyzing the recoil nucleon polarization.

From the measured proton and neutron form factors (and assuming charge symmetry), the $u$ and $d$-quark contributions to the nucleon form factor can be determined. Information on the $s$-quark contribution can be obtained by adding the results of parity violating scattering off the proton (parity violation is due to interference between the exchange of a virtual photon and a $Z^{0}$ ).

To derive neutron form factors from either deuteron or ${ }^{3} \mathrm{He}$ data requires careful consideration of nuclear effects. Observables and kinematical conditions need to be selected to minimize nuclear corrections. Systematic cal-

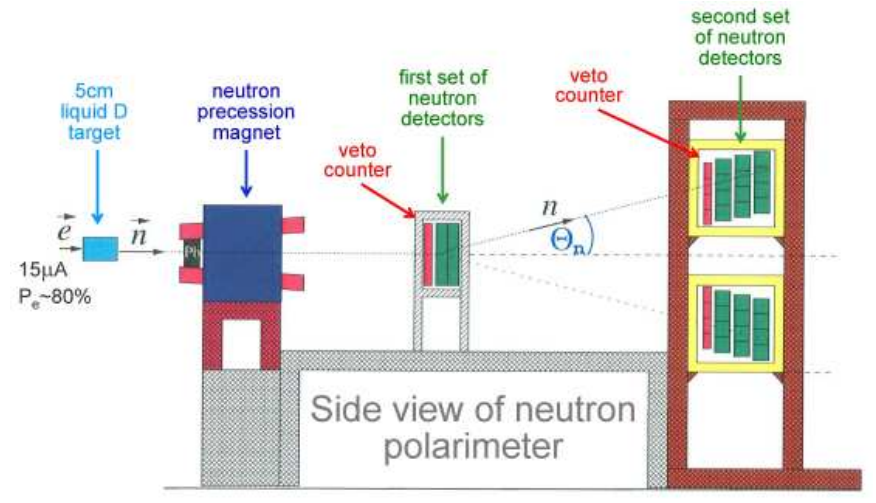

Fig. 13. Neutron polarimeter part of the $G_{e} / G_{m}$ setup.

culations for all observables, including polarization, have been carried out by Arenhövel and collaborators [8].

For example, the calculations show that the asymme$\operatorname{try} A_{e D}^{V}$ in the quasi-free $e-n$ scattering of polarized electrons off a polarized deuterium target (with the deuteron spin perpendicular to the direction of the momentum transfer $Q$ ) is linearly dependent on $G_{e}^{n}$; there is very little background. The remaining sensitivity to the reaction dynamics (final-state interactions, isobar configurations, meson-exchange currents, and relativistic effects) and to the $N N$-potential can be quantified, and corrections can be applied to the measured $G_{e}^{n}$ value.

MAMI has made significant contributions to all nucleon form factors [9]. For the neutron, the ratio $G_{e} / G_{m}$ has been measured in quasi-free $e-n$ kinematics with two different polarization techniques: 1) using polarized electrons off an unpolarized D target and analyzing the recoil neutron polarization, and 2) using polarized electrons on a polarized ${ }^{3} \mathrm{He}$ target. The neutron magnetic form factor, $G_{m}^{n}$, has been determined via the quasi-free $e \mathrm{D} \rightarrow e^{\prime} n(p)$ reaction, and the strange quark contribution has been measured via parity-violating electron scattering off the proton [10].

A particularly interesting experimental technique was developed for measuring the ratio $G_{e}^{n} / G_{m}^{n}$ in the quasi-free $D\left(e, e^{\prime} n\right) p$ scattering of polarized electrons off an unpolarized $D$ target and analyzing the longitudinal and transverse polarization of the recoiling neutron. The setup of the neutron polarimeter is shown in fig. 13. The polarimeter is only sensitive to the transverse polarization components. To access the longitudinal polarization component, a magnet (which also duplicates as a sweeping magnet for charged particles) is used to precess the longitudinal component into the transverse plane. The experimental trick is now to precess the dominant longitudinal component just enough to compensate the small transverse component. By varying the magnetic field and thus the neutron precession angle, a setting can be found where the transverse neutron polarization disappears. $G_{e} / G_{m}$ can then be directly calculated from the precession angle. The great advantage of this null measurement which was used at MAMI for the first time is that it is completely insensitive to the precise 


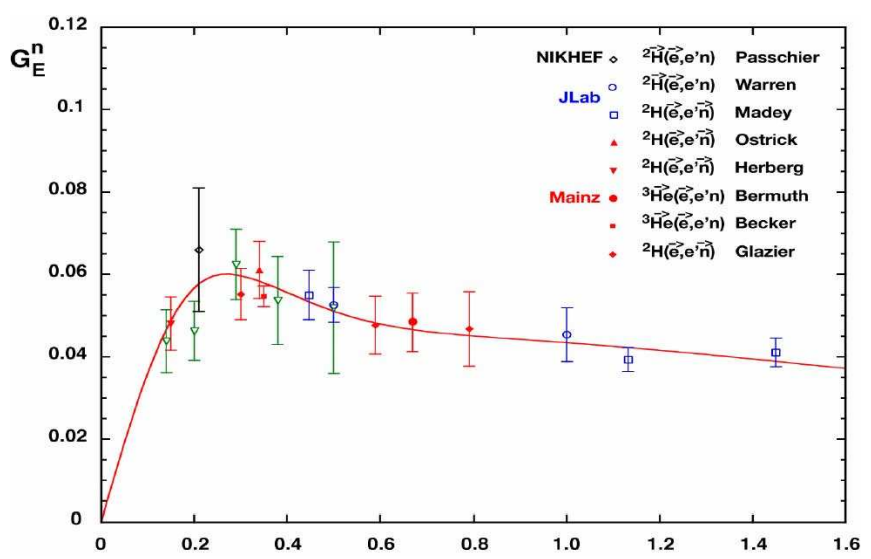

Fig. 14. Neutron electric form factor, $G_{e}^{n}$, as a function of $Q^{2}$ in units of $(\mathrm{GeV} / c)^{2}$. The red curve is the best fit to the world nucleon form factor data [11].

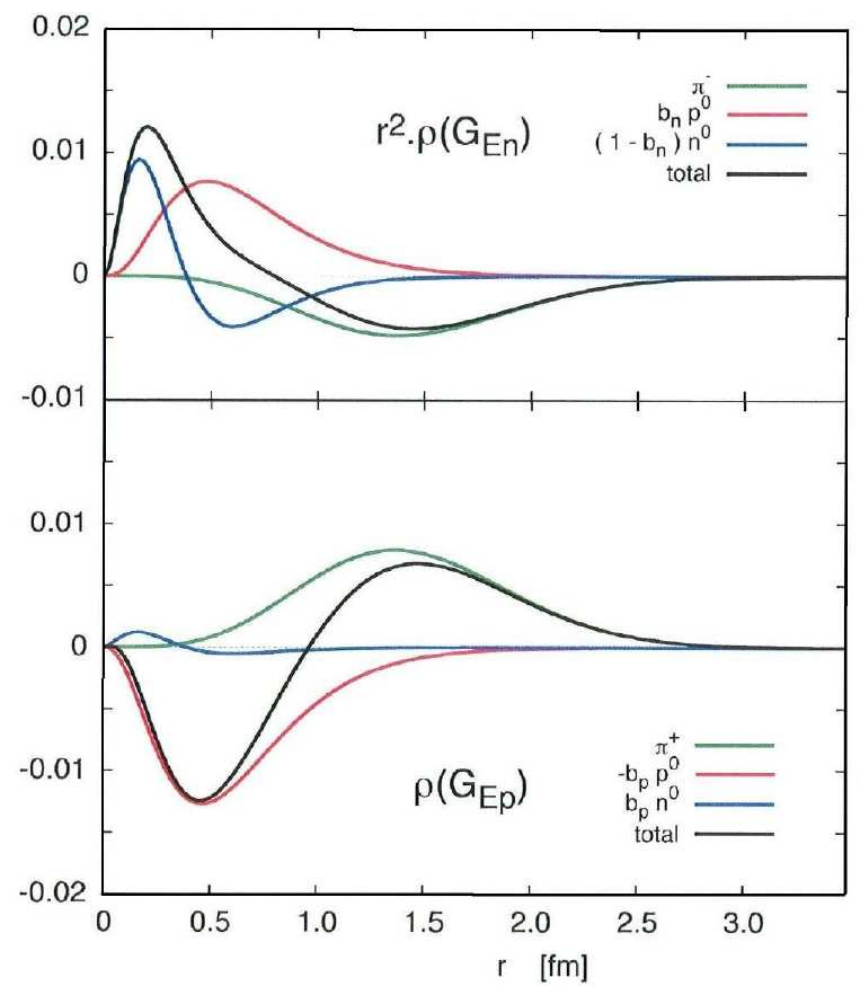

Fig. 15. Neutron (top) and proton (bottom) radial charge distributions from a Fourier analysis of the world nucleon form factor data [11]. The green solid lines show the pion cloud contribution. Note that for the neutron $r^{2} \rho(r)$ is plotted which emphasizes the contributions at large radii.

values of the beam polarization and the analyzing power of the neutron polarimeter.

The MAMI measurements off $\mathrm{D}$ and ${ }^{3} \mathrm{He}$, in combination with measurements from other laboratories, have now led to a consistent set of experimental data for the neutron electric form factor (see fig. 14). No generally accepted theoretical interpretation exists.

Friedrich and Walcher [11] arrived at an intuitive interpretation by describing the nucleon as the sum of a bare

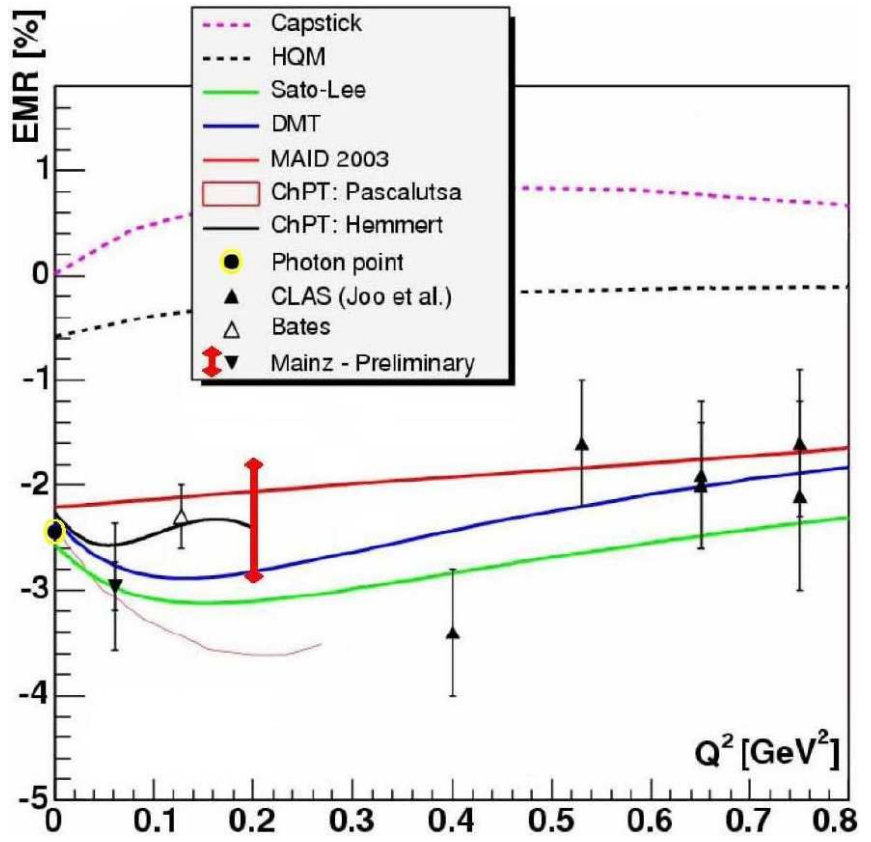

Fig. 16. Ratio of electric quadrupole, $E 2$, to magnetic dipole, $M 1$, strength in the $N \rightarrow \Delta(1232)$ transition as a function of $Q^{2}$.

nucleon and a polarisation part. By fitting all proton and the neutron form factor data, a picture emerges where the neutron spends a considerable part of its time as a system of a proton (located close to the center) and a $\pi^{-}$(located on average about $1.5 \mathrm{fm}$ away from the center); see fig. 15 for the charge distributions of neutron and proton. Similarly, the proton can be viewed as spending part of its time as a neutron- $\pi^{+}$combination.

\subsection{Excitation of the $\Delta(1232)$-resonance}

The physics motivation for studying the electromagnetic excitation of the nucleon resonances is to understand QCD in the strong coupling regime. The mass spectrum and the quantum numbers of the nucleon excited states need to be understood in terms of the relevant degrees of freedom and the wave function and the interaction of the constituents.

The electromagnetic amplitudes for the $N \rightarrow N^{*}$ transition are sensitive to the difference between the quark wave functions of the $N$ and $N^{*}$. The spatial resolution of the probe can be tuned by varying the momentum transfer. The electric and magnetic parts of the transition can be separated using partial-wave analysis (PWA) techniques.

The determination of the electromagnetic transition form factors for the $N \rightarrow N^{*}$ transition requires a large, high-quality data set covering a broad kinematical range in momentum transfer, excitation energy, decay modes $(\pi, \pi \pi, \eta, \rho, \omega)$, and decay angles. Polarization information is especially useful since it is sensitive to the interference between overlapping resonances, or to the interference between a resonance and the background. 


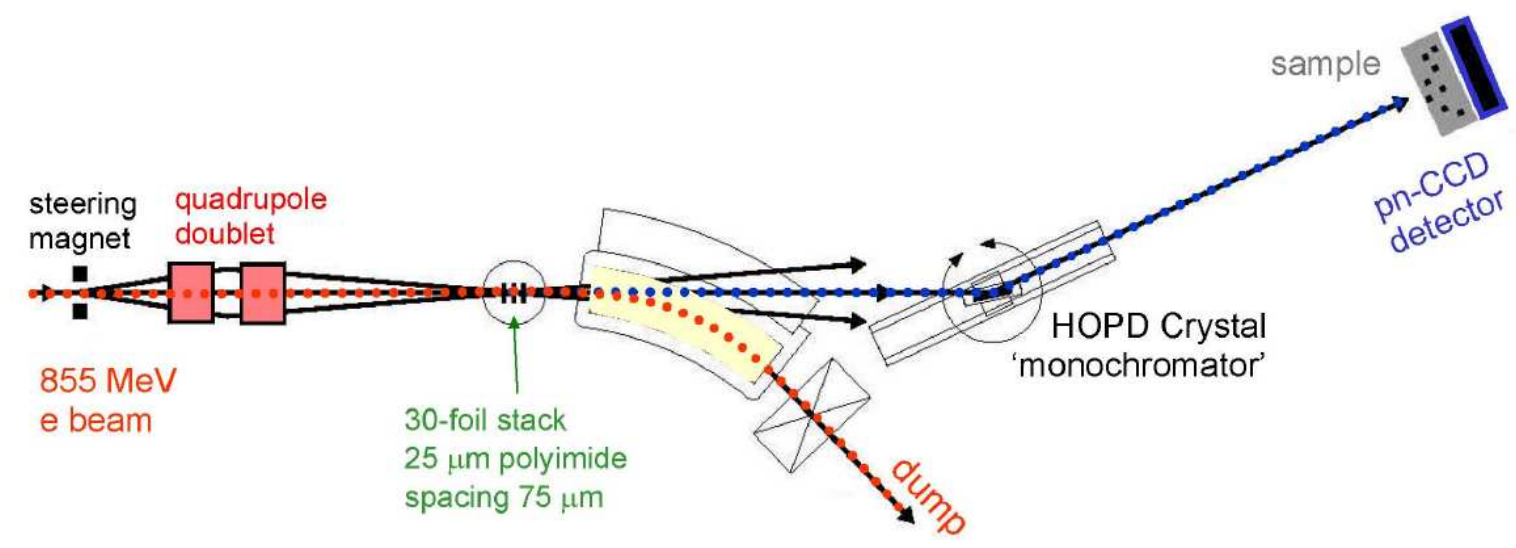

Fig. 17. Setup to use quasi-monochromatic transition radiation for $K$-edge imaging. The sample containing the Mo foil is located in front of the pn-CCD detector.

For higher $N^{*}$ resonances, the extraction of the transition form factor becomes quite involved: in the theoretical description, Born terms, unitarity, and channel coupling need to be taken into account. A full PWA is presently not possible due to lack of data; especially polarization data is missing. To compensate for the lack of experimental data, the analysis is often constrained by assuming the energy dependence of the resonance excitation.

A particularly interesting example is the $N \rightarrow \Delta(1232)$ transition. A full partial wave analysis is possible since the $\Delta(1232)$ is an isolated resonance, and the Watson theorem constrains the phases of the helicity amplitudes. The spin $1 / 2 \rightarrow 3 / 2$ transition is dominated by spin-flip $M 1$; however, non-zero $E 2$ and $C 2$ multipoles are possible. This would be a signature for a non-spherical charge distribution in the $\Delta(1232)$ or could be caused by the virtual photon coupling to a pion cloud.

At MAMI, a major experimental effort $[3,12]$ was launched to determine the $E 2 / M 1$ ratio for the $N \rightarrow \Delta(1232)$ transition starting at the real photon point and extending the measurements to virtual photons. For real photons, the experiments used the tagging system to produce linearly polarized photons and TAPS to detect the $\pi^{0} \rightarrow \gamma \gamma$ decay. For electroproduction, the three-spectrometer setup was used to measure the $e p \rightarrow e^{\prime} p\left(\pi^{0}\right)$ process. Tilting the proton spectrometer provided access to out-of-plane observables.

The $Q^{2}$-dependence of the $E 2 / M 1$ ratio is shown in fig. 16. The results show that the $E 2 / M 1$ ratio is small (around 2\%) and negative. This finding is at variance with all models that consider constituent quarks, only. Models that explicitly include the pion cloud can explain the data.

\subsection{Production of low-energy radiation}

In the context of the applied physics program [13], techniques were developed that make use of the high-quality MAMI electron beam for the production of high brilliance X-rays. In addition to clarifying fundamental aspects of radiation production, there are potential practical applications, e.g. in the material sciences, in biology, and in medicine.

The most powerful X-ray sources in the energy regime of interest $(K$-edge of oxygen at $0.53 \mathrm{keV}$ to the $K$-edge of iodine at $33.16 \mathrm{keV}$ ) are dedicated synchrotron radiation facilities. Modern electron accelerators with their low emittance electron beams may offer an attractive alternative. The interaction of these beams with matter have the potential of producing high brilliance X-rays with a tunable time structure. Processes of interest are transition radiation, parametric X-rays, undulator radiation, the Smith-Purcell effect, and channeling radiation.

An illustrative example is the use of the $855 \mathrm{MeV}$ MAMI beam hitting a stack of 30 polyimide foils to produce hard X-rays via transition radiation [14]. Using a highly oriented pyrolytic graphite crystal, an X-ray beam of about $20 \mathrm{keV}$ was prepared; its two-dimensional spatial distribution was measured in a pn-CCD detector (see fig. 17 for details). Due to the crystal monochromator, the X-ray beam had a correlation between position and energy. By synchronously changing the electron beam direction and the crystal position, the X-ray energy spectrum could be swept as a function of time. This technique avoids making the X-ray beam monochromatic with a slit system, and thus does not reduce the flux. Using the Xray beam for $K$-edge imaging as a demonstration project, a $2.5 \mu \mathrm{m}$ Mo foil hidden in a 100 times thicker copper foil could be detected. Once perfected, this technique may be used to image the human lung using xenon (mixed with oxygen) as an absorber.

\section{Experiment - Theory interplay at MAMI}

As already pointed out in the discussion of the experimental program, there is a very close and effective collaboration between the theorists working in the Institute and the experimentalists using MAMI. This tight coupling increases the impact of the MAMI experimental program in two important ways. 


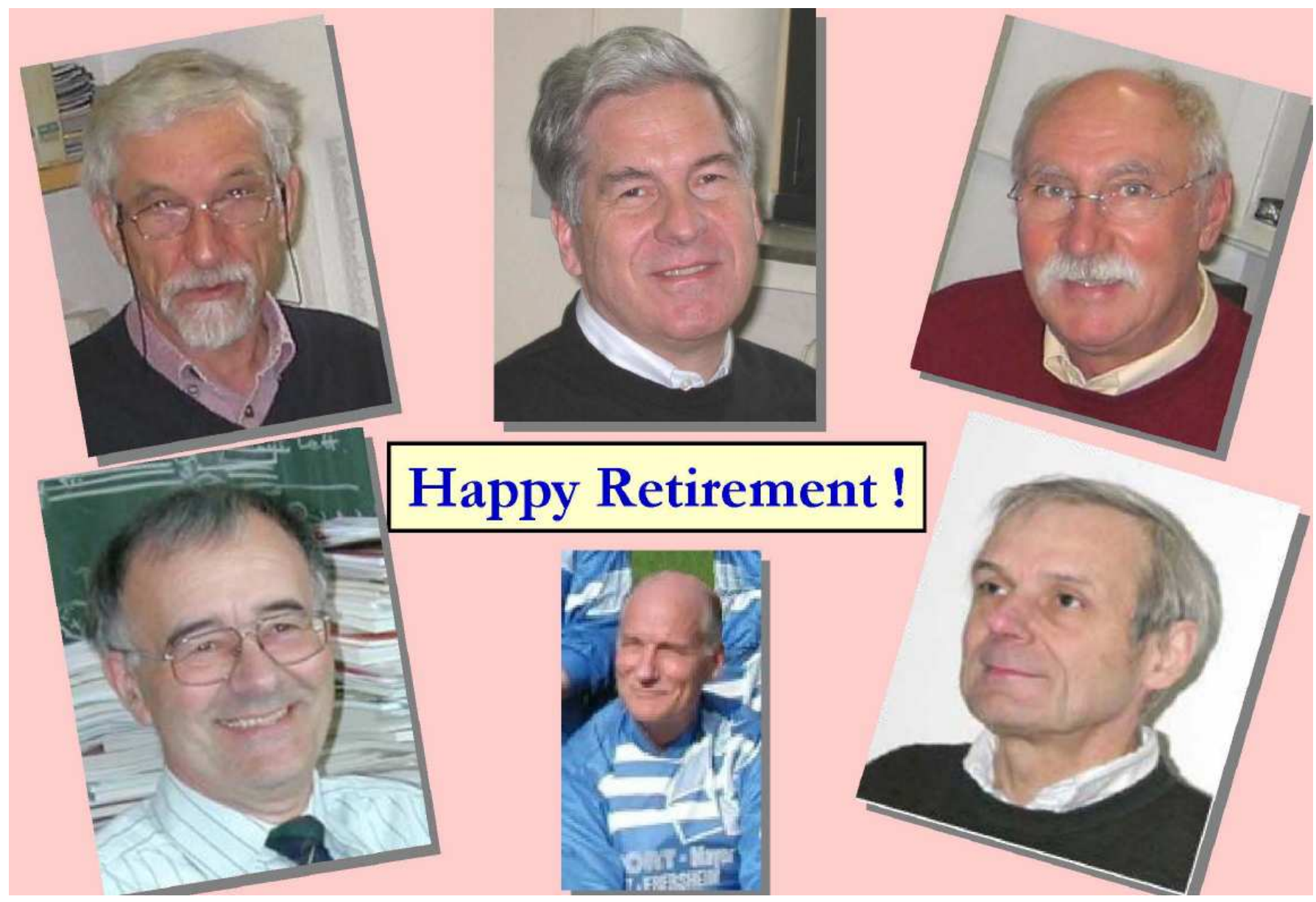

Fig. 18. Photos of the retirees. Top row from left to right: Hartmuth Arenhövel, Thomas Walcher, and Karl-Heinz Kaiser; bottom row: Dieter Drechsel, Jörg Friedrich, and Hartmut Backe.

First, it provides the necessary corrections and facilitates the physics interpretation for quantities for which the measurement strategy is clear and unambiguous. Typical examples are the calculations of the corrections to the measured $G_{e}^{n}$ values, or the corrections to the GDH integral to account for the unmeasured angular and energy range.

Second, theoretical support starting in the early phase of the experiment makes it possible to attack the determination of quantities for which - due to the existence of strong competing channels - the measurement and analysis strategy is not obvious. Typical examples include the extraction of the nucleon polarizability from $\gamma p \rightarrow \gamma p$, the pion polarizability from $\gamma p \rightarrow \pi^{+} n \gamma$, and the magnetic moment of the $\Delta^{+}(1232)$ from $\gamma p \rightarrow p \pi^{0} \gamma$.

The tight coupling between theorists and experimentalists at MAMI is unusual and unique. It clearly has been a major contributor to the success of MAMI. The ingredients necessary for the tight coupling are not easy to identify, and cannot easily be transfered to other facilities. Very likely, the early history of the MAMI project and the personal inclination of the people involved on both sides have played an important role.

\section{MAMI funding and operation}

Securing steady funding for operating a major electromagnetic nuclear physics facility within a university environment has been a real challenge. Contributions from the University of Mainz, the state of Rhineland-Palatinate, and from the Federal Government are required to keep the operation of MAMI financially viable. A large fraction of the federal funding is provided by the DFG in the framework of the SFB which is meant to support new ventures for a limited period of time, but not meant to support steady-state operation. The success of MAMI in this difficult funding environment is testimony to the skills of the people in charge of the Institute.

The MAMI physics program has been comprehensive and more characteristic for a national facility than for a university-based accelerator. No physics problem that was worth attacking has been left out, even when it required additions to the experimental equipment and improvements to the accelerator (e.g.: the parity violation program).

International collaborators have played an important role at MAMI, contributing both ideas and experimental equipment. They obviously felt welcome at the Institute; the natural hospitality and curiosity of the population of 
Mainz and the surrounding areas have likely had a beneficial effect, too.

MAMI has been an ideal training ground for students who could experience all stages of an experiment, from planning to analyzing and publishing the results. The students were also trained in developing and using sophisticated experimental equipment, a good preparation for those who went on to pursue careers outside of nuclear physics. In addition to educating students at MAMI, a generation of young researchers has been trained who have now gone out and successfully competed for faculty positions.

\section{The legacy of 20 year of MAMI physics}

What this 20-year period at MAMI will be remembered for will depend on the range of interests of the person asking the question.

The development of the single-sided microtrons, MAMI A and B, has already changed the textbooks on accelerators; the design and construction of the doublesided MAMI C microtron will complete the microtron development.

The quality of the experimental data will not be surpassed for a long time. Note that very often the accuracy of the final answer is limited by the accuracy of the theoretical corrections. Better accuracy can only be achieved by an improvement of both the experimental results and the theoretical interpretation.

Twenty years of MAMI physics have demonstrated that a tight coupling between theory and experiment can be mutually beneficial. This coupling, which may not be easy to reproduce at other facilities, has been a major contributor to the success of MAMI.

Finally, 20 years of MAMI physics have demonstrated that it is possible - although with a lot of effort- to operate a major facility within the framework of a German university.

\section{Summary}

The development of the physics program and the experimental facilities at the Mainz Microtron MAMI over the last twenty years has been reviewed. MAMI and its user community have been working at the forefront of electromagnetic nuclear physics. Novel electron accelerators and experimental equipment were developed and have been used for ground-breaking research into the structure of nucleons and nuclei.

The six people, Hartmuth Arenhövel, Hartmut Backe, Dieter Drechsel, Jörg Friedrich, Karl-Heinz Kaiser, and Thomas Walcher (fig. 18) who have retired (or are about to retire) have played key roles in this development. They have every right to be proud of what has been accomplished. I would like to take this opportunity to wish them "Happy Retirement".

\section{References}

1. A. Jankowiak, these proceedings.

2. A. Jankowiak et al., prepared for the 8th European Particle Accelerator Conference (EPAC 2002), Paris, France, 3-7 June 2002.

3. R. Beck, these proceedings.

4. M. Vanderhaeghen, these proceedings.

5. N. d'Hose, these proceedings.

6. R. Young (Jefferson Lab), private communication.

7. A. Thomas, these proceedings.

8. H. Arenhövel et al., Z. Phys. A 331, 123 (1988).

9. M. Ostrick, these proceedings.

10. F. Maas, these proceedings.

11. J. Friedrich, T. Walcher, Eur. Phys. J. A 17, 607 (2003), arXiv:hep-ph/0303054.

12. H. Schmieden, these proceedings.

13. W. Lauth, these proceedings.

14. F. Hagenbuck et al., IEEE Trans. Nucl. Sci. 48, 843 (2001). 